\title{
Correction to: A diketopyrrolopyrrole-based nonfullerene acceptor for organic solar cells with a high open-circuit voltage of $1.17 \mathrm{~V}$
}

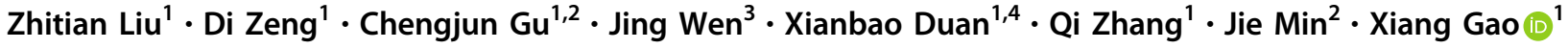

Published online: 26 September 2019

(C) The Society of Polymer Science, Japan 2019

\section{Correction to: Polymer Journal}

$$
\text { https://doi.org/10.1038/s41428-019-0197-0 }
$$

The authors noted that there was one grant number wrong in Acknowledgement. The grant number of the Natural
Science Foundation of Hubei was originally given as 'ZRMS2017001'. This should have read as '2017CFB288'.
Xiang Gao

xgao@wit.edu.cn

1 Institute of Materials for Optoelectronics and New Energy, School of Material Science \& Engineering, Wuhan Institute of Technology, 430073 Wuhan, PR China

2 The Institute for Advanced Studies, Wuhan University, 430073 Wuhan, China

3 School of Chemistry and Chemical Engineering, Nanjing University, 210023 Nanjing, PR China

4 School of Material Science \& Engineering, Huazhong University of Science and Technology, 430073 Wuhan, China 